\title{
ARE PRIMITIVE WORDS UNIVERSAL FOR INFINITE SYMMETRIC GROUPS?
}

\author{
BY
}

D. M. SILBERGER

\begin{abstract}
Let $W=W\left(x_{1}, \ldots, x_{j}\right)$ be any word in the $j$ free generators $x_{1}, \ldots, x_{j}$, and suppose that $W$ cannot be expressed in the form $W=V^{k}$ for $V$ a word and for $k$ an integer with $|k| \neq 1$. We ask whether the equation $f=W$ has a solution $\left(x_{1}, \ldots, x_{j}\right)=\left(a_{1}, \ldots, a_{j}\right) \in G^{j}$ whenever $G$ is an infinite symmetric group and $f$ is an element in $G$. We establish an affirmative answer in the case that $W(x, y)=x^{m} y^{n}$ for $m$ and $n$ nonzero integers.
\end{abstract}

1. Introduction. $F$ denotes the free group generated by the $j$ distinct free generators $x_{1}, \ldots, x_{j}$; and $W$ denotes an arbitrary element in $F$.

For $g \in G$ where $G$ is a group, we say that the $j$-tuple $\left(a_{1}, \ldots, a_{j}\right) \in G^{j}$ is a solution in $G$ for the equation $g=W$ iff $h(W)=g$ where $h: F \rightarrow G$ is the homomorphism generated by the function $x_{1} \mapsto a_{1}, \ldots, x_{j} \mapsto a_{j}$. When $g=W$ has such a solution $\left(a_{1}, \ldots, a_{j}\right)$ then we write $g=W\left(a_{1}, \ldots, a_{j}\right)$, and we say that the equation $g=W$ is solvable in $G$.

We say that $W$ is universal for $G$ iff $g=W$ is solvable in $G$ for every $g \in G$. For a family $S$ of groups we say that $W$ is $S$-universal iff $W$ is universal for every $G \in S$.

For $X$ a set, $S_{X}$ denotes the symmetric group on $X$, and $A_{X}$ denotes the alternating subgroup of $S_{X}$ when $X$ is finite. ISym denotes the family of all infinite symmetric groups.

Recent papers $[6,7,12,13,14]$ are addressed to the task of characterizing, for various families $S$ of groups, the set of $S$-universal words. The question serving as the title of the present paper suggests a special role in such studies for the family ISym. We now clarify this titular question.

$W$ is said to be group-equivalent to a word $U$ iff there exists a finite sequence $W=V_{0}, V_{1}, \ldots, V_{t}=U$ of words such that for each nonnegative integer $i<t$ one of the following three conditions is satisfied: Either $V_{i}=A B B^{-1} C$ while $V_{i+1}=A C$ for some words $A, B$, and $C$; or $V_{i+1}=A B^{-1} B C$ while $V_{i}=A C$ for some words $A, B$, and $C$; or $V_{i+1}=A^{-1} V_{i} A$ for some word $A$.

$\lambda(W)$ denotes the sum of the absolute values of the exponents of the letters in $W$. Concording with $[12, \S 2]$ we say that $W$ is cyclically reduced iff $\lambda(W) \leqslant \lambda(U)$ for every $U$ which is group-equivalent to $W$.

Received by the editors June 4, 1981 and, in revised form, March 15, 1982.

1980 Mathematics Subject Classification. Primary 20B30, 20F10; Secondary 03D40.

${ }^{1}$ This research was partially supported by FINEP and by CNPq. 
For cyclically reduced words $W$ and $U$ it is an exercise to prove that $W$ is group-equivalent to $U$ if and only if there exist words $A$ and $B$ such that $W=A B$ while $U=B A$.

$W$ is said to be a power iff the equality $W=V^{n}$ is satisfied for some word $V$ and for some integer $n$ with $|n| \neq 1$. We call a word $U$ primitive iff no power is group-equivalent to $U$. Precisely formulated our titular question now becomes:

Is every primitive word ISym-universal?

Henceforth $x=x_{1}$ and $y=x_{2}$. In [6], Proposition 2(i) asserts that the word $x^{2} y^{2}$ is ISym-universal. Our main contribution is the following generalization.

THEOREM 1. For $m$ and $n$ nonzero integers the word $x^{m} y^{n}$ is ISym-universal.

In $\S 2$ we formalize truisms which facilitate our proof in $\$ 3$ of Theorem 1 . In $\$ 4$ we close with a brief survey. But first we delineate for our titular question its boundaries of interest.

Henceforth $Z$ denotes the set of all integers, and $s$ denotes the cyclic permutation $i \mapsto i+1$ of $Z$. By $\operatorname{deg}_{W}\left(x_{i}\right)$ we mean the sum of the exponents of the letter $x_{i}$ in the word $W$. By $\operatorname{gcf}(W)$ we mean the greatest common factor of the integers $\operatorname{deg}_{W}\left(x_{1}\right), \ldots, \operatorname{deg}_{W}\left(x_{j}\right)$.

The thrust of Propositions 2 and 3 below is that it suffices to study cyclically reduced, primitive words $W$ for which $\operatorname{gcf}(W) \neq 1$. We omit the easy proofs of

Proposition 2. Let $n$ be an integer with $|n| \neq 1$.

(1) The equation $s=W^{n}$ is not solvable in $S_{Z}$, and therefore the word $W^{n}$ is not ISym-universal.

(2) If $\operatorname{gcf}(W)=1$, then $W$ is universal for every group.

Proposition 3. Let $G$ be a group, let $g \in G$, and let $U$ and $W$ be words with $U$ group-equivalent to $W$. Then the equation $g=W$ is solvable in $G$ if and only if the equation $g=U$ is solvable in $G$.

Proof. It obviously suffices to show that if $g=W$ is solvable in $G$ then $g=V^{-1} W V$ is solvable in $G$ for the arbitrary word $V$. So, let $\left(a_{1}, \ldots, a_{j}\right)$ be a solution in $G$ for the equation $g=W$. For each positive integer $i \leqslant j$ let $b_{i}$ denote the element $V\left(a_{1}, \ldots, a_{j}\right) a_{i} V\left(a_{1}, \ldots, a_{j}\right)^{-1}$ in $G$. Note that the equality

$$
P\left(b_{1}, \ldots, b_{j}\right)=V\left(a_{1}, \ldots, a_{j}\right) P\left(a_{1}, \ldots, a_{j}\right) V\left(a_{1}, \ldots, a_{j}\right)^{-1}
$$

is satisfied for every word $P$. Thus an easy calculation establishes the equality

$$
V\left(b_{1}, \ldots, b_{j}\right)^{-1} W\left(b_{1}, \ldots, b_{j}\right) V\left(b_{1}, \ldots, b_{j}\right)=W\left(a_{1}, \ldots, a_{j}\right) .
$$

Therefore $\left(b_{1}, \ldots, b_{j}\right)$ is a solution in $G$ for the equation $g=V^{-1} W V$.

Proposition 2(2) suggests that there is no dearth of ISym-universal words. On the other hand, Propositions 2(1) and 3 imply that if $W$ is ISym-universal then $W$ is primitive. Propositions 2(1) and 3 together with Theorem 1 establish that the word $x^{k} y^{m} x^{n}$ is ISym-universal if and only if either $k+n \neq 0$ or $|m|=1$. Thus our titular question has an affirmative answer for all words whose 'complexities' are less than four.

Is the word $x^{m} y^{n} x^{p} y^{q}$ ISym-universal when it is primitive? 
2. Preliminaries. Henceforth $X$ is an arbitrary set; $\omega$ denotes the set of nonnegative integers; for $0 \neq n \in \omega$ the symbol $n$ denotes also the $n$-membered set $\{0, \ldots, n-1\}$, while 0 denotes also the empty set $\varnothing$. And $W$ is an arbitrary element in the free semigroup $F$.

Let $H \subseteq S_{X}$. Then $H$ is said to be pairwise disjoint in the sense of permutations, abbreviated pdsp, iff whenever $f$ and $g$ are distinct elements in $H$ then $f(t) \neq t$ implies $g(t)=t$ for every $t \in X$. It is easy to see that if $H$ is pdsp, and if $f$ and $g$ are elements in $H$, then $f g=g f$.

We call $f$ a cycle in $X$ iff $f$ is a permutation of $X$ for which there exists $t \in X$ such that $f(t) \neq t$ but $f(v)=v$ for every $v \in X \backslash\left\{f^{i}(t): i \in Z\right\}$. By the length of a cycle $f$ we mean the cardinal number of the set $\left\{f^{i}(t): i \in Z\right\}$ when $f(t) \neq t$. When $f$ is a cycle of infinite length then we say that $f$ is an $\omega$-cycle. When $f$ is a cycle of length $k$ with $1<k \in \omega$, then we say that $f$ is a $k$-cycle. When $f$ is an $\omega$-cycle then we may write $f$ in the form $f=\left(\cdots f^{-1}(t) t f(t) f^{2}(t) \cdots\right)$, given that $f(t) \neq t$. When $f$ is a $k$-cycle for $k \in \omega$ then we may write $f$ in the familiar form $f=\left(t f(t) \cdots f^{k-1}(t)\right)$, given that $f(t) \neq t$.

For each $f \in S_{X}$ there is a unique pdsp set $C_{X}(f)$ of cycles in $X$ such that, for every $t \in X$ with $t \neq f(t)$, there is exactly one element $g \in C_{X}(f)$ such that $f(t)=g(t)$; but, for every $t \in X$ with $t=f(t)$, it happens that $t=h(t)$ for every $h \in C_{X}(f)$. The elements in $C_{X}(f)$ are called cyclic components of $f$. For $f$ and $g$ permutations of $X$ it is evident that $f=g$ if and only if $C_{X}(f)=C_{X}(g)$.

For $f \in S_{X}$ the expression $X £ f$ denotes the support $\{t: f(t) \neq t \in X\}$ in $X$ of $f$.

A permutation $f$ of $X$ is called cyclic iff both $C_{X}(f)=\{f\}$ and $X £ f=X$. It is obvious that the symmetric group $S_{X}$ contains a cyclic permutation if and only if $X$ is a countable set containing more than one distinct element.

LEMMa 4. Let $P$ be a partition of $X$. For every $Y \in P$ let $f_{Y}$ be an element in $S_{Y}$ such that the equation $f_{Y}=W$ has a solution $\left(a_{1 Y}, \ldots, a_{j Y}\right)$ in $S_{Y}$. Let $f=\bigcup\left\{f_{Y}: Y \in P\right\}$, and for each positive integer $i \leqslant j$ let $a_{i}=\cup\left\{a_{i Y}: Y \in P\right\}$. Then of course $f \in S_{X}$, and $f_{Y}=f \uparrow Y$ for every $Y \in P$. Furthermore, $\left(a_{1}, \ldots, a_{j}\right)$ is a solution in $S_{X}$ for the equation $f=W$.

Proof. It is obvious that $\left\{f, a_{1}, \ldots, a_{j}\right\} \subseteq S_{X}$. Let $(p, q) \in f$. Then there is a unique $Y \in P$ such that $p \in Y$. It follows that $(p, q) \in f_{Y}=W\left(a_{1 Y}, \ldots, a_{j Y}\right) \subseteq$ $W\left(a_{1}, \ldots, a_{j}\right)$, and hence that $f \subseteq W\left(a_{1}, \ldots, a_{j}\right)$. But both $f$ and $W\left(a_{j}, \ldots, a_{j}\right)$ are permutations of $X$. The lemma follows.

Lemma 5. Let $X$ be infinite. Let $Y$ be a set with $0<|Y| \leqslant|X|$. Then there is a partition $P$ of $X$ such that $|A|=|Y|$ for every $A \in P$.

Proof. Since $|X|=|X \times Y|$ there is a bijection $\beta: X \rightarrow X \times Y$. For each $z \in X$ let $A_{z}$ denote the set $\{t: \beta(t) \in\{z\} \times Y\}$. Let $P=\left\{A_{z}: z \in x\right\}$.

COROLlaRY 6. $W$ is universal for $S_{Z}$ if and only if $W$ is ISym-universal.

Proof. Let $X$ be infinite, let $f \in S_{X}$, and let $W$ be universal for $S_{Z}$. If $|X £ f| \leqslant|Z|$ then there exists $Y$ such that $X £ f \subseteq Y \subseteq X$ while $|Y|=|Z|$. Hence $f \uparrow Y \in S_{Y}$, and $f \uparrow Y=W\left(a_{1 Y}, \ldots, a_{j Y}\right)$ for some $\left(a_{1 Y}, \ldots, a_{j Y}\right) \in S_{Y}^{j}$. For each positive integer $i \leqslant j$ 
let $a_{i}=a_{i Y} \cup\{(t, t): t \in X \backslash Y\}$. Then $\left(a_{1}, \ldots, a_{j}\right) \in S_{X}^{j}$ and $f=W\left(a_{1}, \ldots, a_{j}\right)$. Therefore we may suppose that $|Z|<|X £ f|$. It follows that $|Z|<\left|C_{X}(f)\right|$, since $|X £ h| \leqslant|Z|$ for every $h \in C_{X}(f)$. Thus by Lemma 5 there exists a partition $P$ of $C_{X}(f)$ such that $|A|=|Z|$ for every $A \in P$.

For each $A \in P$ let $f_{A}$ be the unique permutation of $X$ for which $C_{X}\left(f_{A}\right)=A$, and let $g_{A}$ denote $f_{A} \uparrow X £ f_{A}$. Evidently $\left|X £ f_{A}\right|=|Z|$, and $g_{A}$ is a permutation of the set $X £ f_{A}$. Therefore, there exist permutations $b_{1 A}, \ldots, b_{j A}$ of $X £ f_{A}$ such that $g_{A}=$ $W\left(b_{1 A}, \ldots, b_{j A}\right)$. Let $Y=X \backslash X £ f$, and let $b_{i Y}$ be the identity function on $Y$ for every positive integer $i \leqslant j$; note that $f \nmid Y=W\left(b_{1 Y}, \ldots, b_{j Y}\right)$. Since the family $\{Y\} \cup$ $\left\{X £ f_{A}: A \in P\right\}$ is a partition of the set $X$, the present corollary follows by Lemma 4 .

LEMMA 7. Let $G$ be a group. Then the following two assertions are equivalent:

(1) The word $x^{p} y^{q}$ is universal for $G$ whenever $p$ and $q$ are positive integers.

(2) The word $x^{m} y^{n}$ is universal for $G$ whenever $m$ and $n$ are nonzero integers.

Proof. Suppose the assertion of Lemma 7(1) to be true, and let $f \in G$. If for positive integers $p$ and $q$ there exist elements $a$ and $b$ in $G$ such that $f=a^{p} b^{q}$, then we also have that $f=\left(a^{-1}\right)^{-p} b^{q}=\left(a^{-1}\right)^{-p}\left(b^{-1}\right)^{-q}=a^{p}\left(b^{-1}\right)^{-q}$. Lemma 7(2) follows.

3. A proof of Theorem 1. Until the end of the present section the symbols $m$ and $n$ denote arbitrary positive integers, and $f$ denotes an arbitrary permutation of $Z$.

LEMMA 8. Let $p$ and $k-1$ be positive integers. For each $i \in p$ let $g_{i}$ be a $k$-cycle in $X$ so that $\left\{g_{i}: i \in p\right\}$ is a p-membered set which is pdsp. Then there exists a pk-cycle $g$ in $X$ such that $C_{X}\left(g^{p}\right)=\left\{g_{i}: i \in p\right\}$.

Proof. For each $i \in p$ we write $g_{i}=\left(u_{0}^{i} u_{1}^{i} \cdots u_{k-1}^{i}\right)$. Let $g$ be the $p k$-cycle $\left(u_{0}^{0} u_{0}^{1} \cdots u_{0}^{p^{-1}} u_{1}^{0} u_{1}^{1} \cdots u p^{-1} \cdots u_{k-1}^{0} u_{k-1}^{1} \cdots u_{k-1}^{p-1}\right)$ in $X$.

The construction employed in the foregoing proof we call "the interdigitation, of the $p$-block $g_{0} g_{1} \cdots g_{p-1}$ of $k$-cycles, which yields the $p k$-cycle $g$ ". Of course, in the familiar notation, $g^{p}=g_{0} g_{1} \cdots g_{p-1}$.

COROLlary 9. Let $p$ and $k-1$ be positive integers, and let $K$ be the set of all $k$-cycles in $X$. Let $I$ be a set which is either infinite or whose cardinal number is a multiple of $p$. Let the function $I \rightarrow K$ defined by $i \mapsto g_{i}$ be injective and such that $\left\{g_{i}\right.$ : $i \in I\}$ is pdsp. Then there is a permutation $g$ of $X$ such that every element in $C_{X}(g)$ is a pk-cycle, and such that $C_{X}\left(g^{p}\right)=\left\{g_{i}: i \in I\right\}$.

Proof. There is a partition $L$ of the set $\left\{g_{i}: i \in I\right\}$ into $p$-membered sets. For each $H \in L$, we order the elements of $H$ into a $p$-block, and interdigitate them in order to obtain a $p k$-cycle $G_{H}$ for which $C_{X}\left(G_{H}^{P}\right)=H$. Let $g$ be the permutation of $X$ for which $C_{X}(g)=\cup\left\{C_{X}\left(G_{H}\right): H \in L\right\}$, and note that $C_{X}\left(g^{p}\right)=\left\{g_{i}: i \in I\right\}$.

In reference to Corollary 9 , the cardinal number $|I|$ is less than the cardinal number of the set of all $g$ which satisfy the corollary.

It is perfectly natural to interdigitate $p$-blocks of $\omega$-cycles. The result is always one or more $\omega$-cycles. For instance, by interdigitating the 3-block $h_{0} h_{1} h_{2}$, where $h_{i}$ is the 
$\omega$-cycle $(\cdots i-6 i-3 i i+3 i+6 \cdots)$, we obtain $s$. Clearly $s^{3}=h_{0} h_{1} h_{2}$. We omit the analogous formalities.

LEMMA 10. Let $1<k \in \omega$, and let $f$ be a $k$-cycle in $Z$. Then the equation $f=x^{m} y^{n}$ is solvable in $S_{Z}$.

Proof. Since the set $\{t: f(t)=t\}$ is infinite, there exists an infinite injective sequence $g_{0}, g_{1}, \ldots$ of $k$-cycles in $Z$ such that $\{f\} \cup\left\{g_{i}: i \in \omega\right\}$ is pdsp. Arrange the elements in $\left\{g_{i}: i \in \omega\right\}$ into $n$-blocks, and interdigitate them in order to obtain the $k n$-cyclic components of a permutation $b^{-1}$ such that $C_{Z}\left(b^{-n}\right)=\left\{g_{i}: i \in \omega\right\}$. Arrange the elements in $\{f\} \cup\left\{g_{i}: i \in \omega\right\}$ into $m$-blocks, and interdigitate them in order to obtain the $k m$-cyclic components of a permutation $a$ such that $C_{Z}\left(a^{m}\right)=\{f\} \cup\left\{g_{i}\right.$ : $i \in \omega\}$. Then $f b^{-n}=a^{m}$, whence $f=a^{m} b^{n}$.

COROllary 11. Let $\{t: f(t)=t\}$ be infinite. Then the equation $f=x^{m} y^{n}$ is solvable in $S_{Z}$.

Proof. For each countable cardinal number $k>1$ let $D_{k}$ be the set of all $k$-cyclic

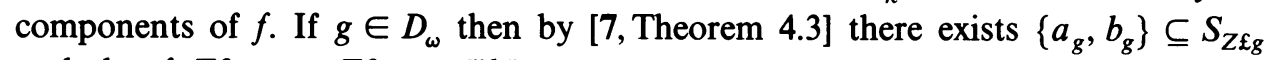
such that $f \uparrow Z £ g=g \uparrow Z £ g=a_{g}^{m} b_{g}^{n}$.

Partition $\{t: f(t)=t\}$ into a family $\left\{P_{k}: 1<k \in \omega\right\}$ of infinite sets. For each finite $k>1$ let $E_{k}$ be the set $P_{k} \cup Z D_{k}$, where $Z D_{k}=\cup\left\{Z £ g: g \in D_{k}\right\}$. Let $d_{k}$ be the permutation such that $C_{Z}\left(d_{k}\right)=D_{k}$. Now, since $P_{k}$ is infinite, there exists an injective infinite sequence $d_{k}^{0}, d_{k}^{1}, \ldots$ of $k$-cycles in $E_{k}$ such that $\left\{d_{k} \uparrow E_{k}\right\} \cup\left\{d_{k}^{i}\right.$ : $i \in \omega\}$ is pdsp. Arrange the $d_{k}^{i}$ into $n$-blocks, and interdigitate in order to obtain the $n k$-cyclic components of a permutation $b_{k}^{-1}$ of $E_{k}$ such that $C_{L}\left(b_{k}^{-n}\right)=\left\{d_{k}^{i}: i \in \omega\right\}$, where $L=E_{k}$. Similarly, arrange the elements of $C_{L}\left(d_{k} \mid E_{k}\right) \cup\left\{d_{k}^{i}: i \in \omega\right\}$ into $m$-blocks, in order to obtain the components, each of which is an $m k$-cycle, of a permutation $a_{k}$ of $E_{k}$ such that $C_{L}\left(a_{k}^{m}\right)=C_{L}\left(d_{k} \mid L\right) \cup\left\{d_{k}^{i}: i \in \omega\right\}$. Note that $a_{k}^{m}=\left(d_{k} \uparrow E_{k}\right) b_{k}^{-n}$, and hence that $f \uparrow E_{k}=d_{k} \uparrow E_{k}=a_{k}^{m} b_{k}^{n}$.

Since $\left\{E_{k}: 1<k \in \omega\right\} \cup\left\{Z £ g: g \in D_{\omega}\right\}$ is a partition of $Z$, the present corollary now follows by Lemma 4.

LEMMA 12. Let $g$ be an w-cycle in $X$. Let $p_{0}, p_{1}, \ldots$ and $q_{0}, q_{1}, \ldots$ be two infinite sequences of integers greater than 1 . Then there exist infinite injective sequences $u_{0}$, $u_{1}, \ldots$ and $v_{0}, v_{1}, \ldots$ satisfying the following six conditions:

(1) $u_{i}$ is a $p_{i}$-cycle in $X$ for every $i \in \omega$.

(2) $v_{i}$ is a $q_{i}$-cycle in $X$ for every $i \in \omega$.

(3) $\left\{u_{i}: i \in \omega\right\}$ is pdsp.

(4) $\left\{v_{i}: i \in \omega\right\}$ is pdsp.

(5) $X \mathfrak{£} u_{i} \cup X \mathfrak{f} v_{i} \subseteq X £ g$ for every $i \in \omega$.

(6) $g=u v$ where $C_{X}(u)=\left\{u_{i}: i \in \omega\right\}$ and where $C_{X}(v)=\left\{v_{i}: i \in \omega\right\}$.

Proof. Our argument generalizes the trick suggested by the diagram in [6]. For each $z \in \omega$ we define $Q_{z}=\sum_{i=0}^{z-1} q_{i}$ and $P_{z}=\sum_{i=0}^{z-1} p_{i}$. 
Since $g$ is an $\omega$-cycle in $X$, we have that $X$ is infinite. Without loss of generality we may suppose that $Z \subseteq X$, that $X £ g=Z$, and that $g \uparrow Z=s$.

For each $i \in \omega$ let $t_{i}=Q_{i}+P_{i}-3 i$, let $v_{i}$ be the $q_{i}$-cycle $\left(t_{i} t_{i}+1 \cdots t_{i}+q_{i}-\right.$ $\left.3 t_{i}+q_{i}-2-i-1\right)$ in $X$; let $y_{i}=Q_{i+1}+P_{i}-3 i-1$, and let $u_{i}$ be the $p_{i}$-cycle $\left(y_{i} y_{i}+1 \cdots y_{i}+p_{i}-3 y_{i}+p_{i}-2-i-1\right)$ in $X$. A routine consideration of cases determined by inequalities establishes that the sequences $u_{0}, u_{1}, \ldots$ and $v_{0}, v_{1}, \ldots$ satisfy the requirements of the lemma.

COROLlARY 13. Let $f$ have at most finitely many cyclic components of finite length. Then the equation $f=x^{m} y^{n}$ is solvable in $S_{Z}$.

Proof. By Corollary 11 we may suppose that the set $\{t: f(t)=t\}$ is not infinite. It follows that $f$ has at least one $\omega$-cyclic component $g$. Let $Y$ denote

\section{$Z £ g \cup \bigcup\{Z £ r: r$ is a finite-cyclic component of $f\}$.}

Observe that every element in $C_{Z \backslash Y}(f \uparrow(Z \backslash Y))$ is an $\omega$-cycle in $Z \backslash Y$. Therefore, by [7, Theorem 4.3] together with Lemma 4, there exist permutations $a_{\omega}$ and $b_{\omega}$ of $Z \backslash Y$ such that $f \uparrow(Z \backslash Y)=a_{\omega}^{m} b_{\omega}^{n}$.

By hypothesis $f$ has exactly $p$ finite-cyclic components $f_{0}, f_{1}, \ldots, f_{p-1}$ for some $p \in \omega$. For each $i \in p$ there is an integer $k_{i}>1$ such that $f_{i}$ is a $k_{i}$-cycle in $X$. In the case that $p=0$ the corollary follows by an argument like that in the preceding paragraph. Therefore we may suppose that $p>0$.

Let $1<q \in \omega$. Then by Lemma 12 there exist two injective sequences $u_{0}, u_{1}, \ldots$ and $v_{0}, v_{1}, \ldots$ having the following four properties:

One. $u_{z p+i}$ is a $k_{i}$-cycle in $Y$ for every $(z, i) \in \omega \times p$.

Two. $v_{i}$ is a $q$-cycle in $Y$ for every $i \in \omega$.

Three. Both of the sets $\left\{f_{i} \uparrow Y: i \in p\right\} \cup\left\{u_{z}: z \in \omega\right\}$ and $\left\{v_{i}: i \in \omega\right\}$ are pdsp.

Four. $g \mid Y=u v$, where $u$ and $v$ are defined by $C_{Y}(u)=\left\{u_{i}: i \in \omega\right\}$ and by $C_{Y}(v)=\left\{v_{i}: i \in \omega\right\}$.

For each $i \in p$ let $U_{i}$ denote the infinite pdsp set $\left\{f_{i} \uparrow Y\right\} \cup\left\{u_{z p+i}: z \in \omega\right\}$ of $k_{i}$-cycles in $Y$; arrange the elements in $U_{i}$ into $m$-blocks and interdigitate in order to obtain the components, each of which is an $m k_{i}$-cycle in $Y$, of a permutation $a_{i}$ of $Y$ for which $C_{Y}\left(a_{i}^{m}\right)=U_{i}$. This can obviously be done in such a way that the set $\left\{a_{i}\right.$ : $i \in p\}$ is pdsp. Let $a_{Y}$ denote the permutation $a_{0} a_{1} \cdots a_{p-1}$ of $Y$.

Arrange the elements in the set $\left\{v_{i}: i \in \omega\right\}$ into $n$-blocks, and interdigitate, thus producing the components, each of which is an $n q$-cycle in $Y$, of a permutation $b_{Y}$ of $Y$ for which $C_{Y}\left(b_{Y}^{n}\right)=\left\{v_{i}: i \in \omega\right\}$. Note that

$$
\begin{aligned}
f \uparrow Y & =\left(f_{0} \uparrow Y\right)\left(f_{1} \uparrow Y\right) \cdots\left(f_{p-1} \uparrow Y\right)(g \uparrow Y) \\
& =\left(f_{0} \uparrow Y\right)\left(f_{1} \uparrow Y\right) \cdots\left(f_{p-1} \uparrow Y\right) u v=a_{Y}^{m} b_{Y}^{n}
\end{aligned}
$$

Since $\{Y, Z \backslash Y\}$ is a partition of $Z$, the present corollary now follows by Lemma 4 .

When $t$ is a real number, the expression int $(t)$ denotes the smallest integer $n$ such that $t \leqslant n$. 
LEMMA 14. Let $1<k \in \omega$, and let $g$ be a $k$-cycle in $X$. Then there exists $h$ such that both $h$ and gh are involutions of $X$, such that $\left|C_{X}(h)\right|=\operatorname{int}((k-2) / 2)$, such that $\left|C_{X}(g h)\right|=\operatorname{int}((k-1) / 2)$, and such that $X £ h \subseteq X £ g$.

Proof. We may suppose that $g=(01 \cdots k-1)$. Let $h=(0 k-2)(1 k-3)$ $\cdots(z k-2-z) \in S_{X}$, where $z$ is the largest integer $i$ for which $i \leqslant k-2-i$. Then $z \leqslant(k-2) / 2<z+1$, and thus we have that $z=(k-2) / 2$ if $k$ is even, but that $z=(k-3) / 2$ if $k$ is odd. Therefore, in the case that $k$ is even we have that $\left|C_{X}(h)\right|=z=(k-2) / 2=\operatorname{int}((k-2) / 2)$, while in the case that $k$ is odd we have that $\left|C_{X}(h)\right|=z+1=(k-1) / 2=\operatorname{int}((k-2) / 2)$, with both cases satisfying the claim.

Next, notice that $g h=(0 k-1)(1 k-2) \cdots(z-1 k-z)(z k-z-1) \in S_{X}$. Therefore, since $z \leqslant k-z-2<k-z-1$ we see that $\left|C_{X}(g h)\right|=z+1=$ $\operatorname{int}((k-1) / 2)$ both in the case that $k$ is even and also in the case that $k$ is odd.

Our final lemma depends upon the fact, obvious from Lemma 14, that if $k>2$ then $C_{X}(h) \neq \varnothing \neq C_{X}(g h)$.

LEMMA 15. The word $x^{m} y^{n}$ is universal for $S_{Z}$.

Proof. By the foregoing results in $\S 3$ we may suppose both that $\{t: f(t)=t\}$ is not infinite, and also that $C_{Z}(f)$ contains no infinite cycles. It follows that there is an infinite injective sequence $f_{0}, f_{1}, \ldots$ of finite cycles with $C_{Z}(f)=\left\{f_{i}: i \in \omega\right\}$. There are two cases to consider.

Case 1. The set $\left\{i: f_{i}\right.$ is a 2-cycle $\}$ is infinite. We may suppose that there exists $z \in \omega$ such that $f_{i}$ is a 2-cycle if and only if $i \geqslant z$. For each $i \in z$ there is an involution $h_{i}$ of $Z$ such that $f_{i} h_{i}$ also is an involution, and such that $Z £ h_{i} \subseteq Z £ f_{i}$. Let $h=h_{0} h_{1} \cdots h_{z-1}$. Since $\{h\} \cup\left\{f_{i}: z \leqslant i \in \omega\right\}$ is pdsp, we see that

$$
f=f_{0} f_{1} \cdots f_{z-1} f_{z} \cdots=f_{0} f_{1} \cdots f_{z-1} h h f_{z} \cdots=H H^{\prime},
$$

where $H$ is the involution

$f_{0} f_{1} \cdots f_{z-1} h f_{z} f_{z+2} \cdots f_{z+2 i} \cdots=\left(f_{0} h_{0}\right)\left(f_{1} h_{1}\right) \cdots\left(f_{z-1} h_{z-1}\right) f_{z} f_{z+2} \cdots f_{z+2 i} \cdots$, and where $H^{\prime}$ is the involution $h f_{z+1} f_{z+3} \cdots f_{z+2 i+1} \cdots$. Clearly both $C_{Z}(H)$ and $C_{Z}\left(H^{\prime}\right)$ are infinite sets. We group the elements of $C_{Z}(H)$ into $m$-blocks and interdigitate as usual in order to obtain the $2 m$-cyclic components of a permutation $a$ of $Z$ for which $a^{m}=H$. Similarly we obtain a permutation $b$ of $Z$ for which $b^{n}=H^{\prime}$. As desired, $f=a^{m} b^{n}$.

Case 2. The set $\left\{i: f_{i}\right.$ is a 2-cycle $\}$ is not infinite. This time we suppose that there is a $z \in \omega$ for which $f_{i}$ is a 2-cycle if and only if $i \in z$. Then, for every integer $i \geqslant z$ there is an integer $k_{i}>2$ for which $f_{i}$ is a $k_{i}$-cycle. But by Lemma 14 there is, for each such $i$, an involution $h_{i}$ with $C_{Z}\left(h_{i}\right) \neq \varnothing \neq C_{Z}\left(f_{i} h_{i}\right)$, and such that $f_{i} h_{i}$ also is an involution, and furthermore such that $Z £ h_{i} \subseteq Z £ f_{i}$. It follows that $\left\{h_{i}: z \leqslant i \in \omega\right\}$ is pdsp, and hence that we may define $h$ by

$$
C_{Z}(h)=\bigcup\left\{C_{Z}\left(h_{i}\right): z \leqslant i \in \omega\right\} .
$$

Of course $h$ also is an involution. Furthermore, both $C_{Z}(f h)$ and $C_{Z}(h)$ are infinite collections of 2-cycles. By arranging $C_{Z}(f h)$ into $m$-blocks and interdigitating, we 
obtain a permutation $a$ of $Z$ such that $a^{m}=f h$. By arranging $C_{Z}(h)$ into $n$-blocks, and interdigitating, we obtain a permutation $b$ of $Z$ such that $b^{n}=h$. It follows that $f=f h h=a^{m} b^{n}$.

Theorem 1 is an immediate consequence of Corollary 6 together with Lemmas 7 and 15 .

4. Survey. For $p$ and $q$ integers, $(p: q)$ denotes the greatest common factor of $p$ and $q$. For $f \in S_{X}$ the expression $C_{X}(f ; k)$ denotes the set of all $k$-cyclic components of $f$.

When $W$ is cyclically reduced, then it is evident that $W$ is primitive if and only if $W$ is not a power. The following result sharpens our introductory Proposition 2(1).

Proposition 16. Let $0 \neq n \in Z$. The equation $f=x^{n}$ is solvable in $S_{X}$ if and only if both of the following conditions are satisfied for $f$ where $f \in S_{X}$ :

(1) Either the set $C_{X}(f ; \omega)$ is infinite or the integer $\left|C_{X}(f ; \omega)\right|$ is a multiple of $n$.

(2) For every integer $k>1$ either the set $C_{X}(f ; k /(n: k))$ is infinite, or the integer $\left|C_{X}(f ; k /(n: k))\right|$ is a multiple of $(n: k)$.

Proof. Suppose that $f$ satisfies both of the conditions 16(1) and 16(2). The condition 16(1) implies that the set $C_{X}(f ; \omega)$ can be partitioned into a family $P_{\omega}$ of $|n|$-membered subsets. For each $D \in P_{\omega}$ the elements in the set $D$ can be arranged into an $|n|$-block of $\omega$-cyclic components of $f$, and this block interdigitated in order to obtain an $\omega$-cycle $G_{\omega, D}$ such that $C_{X}\left(G_{\omega, D}^{|n|}\right)=D$. If $n=|n|$ then let $H_{\omega, D}=G_{\omega, D}$, but if $n=-|n|$ then let $H_{\omega, D}=G_{\omega, D}^{-1}$, for each $D \in P_{\omega}$.

Now choose $k>1$. Condition $16(2)$ implies that the set $C_{X}(f ; k /(n: k))$ can be partitioned into a family $P_{k}$ of $(n: k)$-membered subsets. For each $E \in P_{k}$ we arrange the elements of $E$ into an $(n: k)$-block $g_{0}^{E} g_{1}^{E} \cdots g_{(n: k)-1}^{E}$. Since the integers $n /(n: k)$ and $k /(n: k)$ are relatively prime, for each $i \in(n: k)$ there is a cycle $h_{E, i}$ in $X$ of length $k /\left(n:^{\prime} k\right)$ such that $X £ h_{E, i}=X £ g_{i}^{E}$, and such that $h_{E, i}^{n /(n: k)}=g_{i}^{E}$. We interdigitate the $(n: k)$-block $h_{E, 0} h_{E, 1} \cdots h_{E,(n: k)-1^{\prime}}$ and thus produce a $k$-cycle $H_{k, E}$ such that $H_{k, E}^{(n: k)}=h_{E, 0} h_{E, 1} \cdots h_{E,(n: k)-1}$. Then $C_{X}\left(H_{k, E}^{n}\right)=E$.

Finally, define the permutation $a$ of $X$ by the stipulation that

$$
C_{X}(a)=\left\{H_{\omega, D}: D \in P_{\omega}\right\} \cup \bigcup\left\{\left\{H_{k, E}: E \in P_{k}\right\}: 1<k \in \omega\right\} .
$$

Note that $f=a^{n}$.

In order to establish the converse we now suppose that $f=b^{n}$ for some $b \in S_{X}$. Let $g \in C_{X}(b)$. If $g$ is an $\omega$-cycle, then $C_{X}\left(g^{n}\right)$ is a pdsp set of exactly $|n|$ cycles of infinite length. On the other hand, if $1<k \in \omega$, and if $g$ is a $k$-cycle, then it is easy to see that $C_{X}\left(g^{n}\right)$ is a set of exactly $(n: k)$ cycles of length $k /(n: k)$.

If $1<k \in \omega$, and if $n$ is an integer such that $(n: i)=1$ for every positive integer $i \leqslant k$, then the word $x^{n}$ is universal for every $S_{i}$ with $0<i \leqslant k$. Also, if $(n: i)>1$ for some positive integer $i \leqslant k$, then the word $x^{n}$ is not universal for $S_{k}$. Note that $x^{-101}$ is universal for $S_{k}$ whenever $0<k \leqslant 100$, but that $x^{-101}$ fails to be universal for any $S_{k}$ with $100<k$.

The notion, of the 'complexity' of a word, is most easily expressed by means of examples: The words $x, y^{2}$, and $x_{3}^{-7}$ are of complexity one. The words $x y, y^{2} x^{-3}$, and 
$y^{2} x^{2}$ are of complexity two. The words $x^{3} y^{-2} x^{2}, y^{2} x^{-2} y^{2}, x^{2} y^{-2} x^{-2}$, and $x_{1}^{3} x_{3}^{-2} x_{2}^{-5}$ are of complexity three. The word $x y^{-2} x^{3} y^{-1} x^{3} y x^{-1}$ is of complexity seven.

Incidentally, of the eleven words mentioned in the preceding paragraph, all are primitive except for $y^{2}, x_{3}^{-7}, x^{2} y^{-2} x^{-2}$ and $x y^{-2} x^{3} y^{-1} x^{3} y x^{-1}$. The word $x^{2} y^{-2} x^{-2}$ is not primitive because it is group-equivalent to the power $y^{-2}$. The word $x y^{-2} x^{3} y^{-1} x^{3} y x^{-1}$ is not primitive because it is group-equivalent to the power $\left(x^{3} y^{-1}\right)^{2}$. By the way, $\left(x^{3} y^{-1}\right)^{2}$ is of complexity four.

We now consider the possible ISym-universality of words of complexity at least four.

In [15] it is shown that the commutator word $x y x^{-1} y^{-1}$ is ISym-universal. More recent related results are encountered in $[2,5,9]$. Of course $x y x^{-1} y^{-1}$ is primitive, cyclically reduced, of complexity four; and $\operatorname{gcf}\left(x y x^{-1} y^{-1}\right)$ is undefined.

When $1<k \in \omega$, and when $\{a, b\} \subseteq S_{k}$, then $a b a^{-1} b^{-1}$ is an even permutation; therefore $x y x^{-1} y^{-1}$ is not universal for $S_{k}$. On the other hand [3], also referring us in this connection to A. M. Gleason in [10,p. 172], establishes that $x y x^{-1} y^{-1}$ is universal for every alternating group $A_{k}$.

In [4] it is shown that the equation $s=W$ is solvable in $S_{Z}$ for every primitive $W$ whose complexity is less than six; here, once again, $s$ is the successor permutation on $Z$. Obviously, any word $V$ for which the equation $s=V$ is solvable in $S_{Z}$, and which is universal for every finite $S_{k}$, is universal for every symmetric group.

The ISym-universal word $x^{2} y^{2}$, which is universal for no nontrivial finite symmetric group, is known to be universal for every alternating group.

For $f \in S_{k}$ with $0<k \leqslant n$, the equation $f=x^{n !} y^{n !}$ is solvable in $S_{k}$ if and only if $f$ is the identity permutation of the set $k$. But the word $x^{n !} y^{n !}$ is ISym-universal.

For each pair $(p, q)$ of nonzero integers does there exist an integer $N(p, q)$ such that $x^{p} y^{q}$ is universal for every $A_{k}$ with $k>N(p, q)$ ?

Thus far, every effort has been stymied to prove that the equation $s=W$ is solvable in $S_{Z}$ for every primitive $W$, although [1 and 16] have made inroads. In particular it remains unknown whether $s=W$ is solvable in $S_{Z}$ for $W=\left(x^{2} y^{2}\right)^{3} y^{2}$, for $W=x^{3} y^{2} x^{2} y x y$, or for $W=x^{3} y^{2} x y x^{2} y$. Since $\operatorname{gcf}(W)$ is even for each of these recalcitrant words, none of them is universal for any nontrivial finite symmetric group. We do not know for which alternating groups they are universal.

It continues to be our hope that methods of the sort used in $\$ 3$ will establish the ISym-universality of all primitive words of complexity four. But, M. P. Borba has discovered some stumbling blocks: Is the equation $(01)=x^{3} y^{3} x y$ solvable in $S_{Z}$ ? What about $(01)=x^{5} y^{5} x y$ ?

The following results are representative of our partial successes. The first is a slight extension of the, independently achieved, identical [6, Proposition 2(ii)] and [16, Corolário 5.7].

Proposition 17. Let there be exactly two letters $x_{v}$ and $x_{u}$ in $W$ such that $\operatorname{deg}_{W}\left(x_{v}\right)$ and $\operatorname{deg}_{W}\left(x_{u}\right)$ are odd integers. Then $W$ is universal for every symmetric group.

Proof. By Lemmas 12 and 14 we have that every cycle $g$ in $X$ can be expressed in the form $g=h_{1} h_{2}$ for involutions $h_{1}$ and $h_{2}$ of $X$ with $X £ h_{1} \cup X £ h_{2} \subseteq X £ g$. It 
follows that for every permutation $f$ of $X$ there exist involutions $a$ and $b$ of $X$ such that $f=a b$. The proposition is an obvious consequence of this fact.

For the complexity-four case, the foregoing proposition establishes that the word $x^{m} y^{n} x^{p} y^{q}$ is universal for every symmetric group if $m$ and $n$ are even while $p$ and $q$ are odd. We do not know for which alternating groups such words $x^{m} y^{n} x^{p} y^{q}$ are universal.

The proof sketch offered for the following result is a précis of $\S 3$. The class of words, whose ISym-universality it establishes, is obviously more ample than the complexity-four subclass to which we restrict our statement in the interests of simplicity.

Proposition 18. Let $m$ and $n$ be nonzero integers, let $M$ be a multiple of $2 m$, and let $N$ be a multiple of $2 n$. Then the word $x^{M} y^{N} x^{m} y^{n}$ is ISym-universal.

Proof SKeTCH. Let $f \in S_{Z}$. The proposition is established when it has been shown that there exist involutions $u$ and $v$ of $Z$, each of which has infinitely many 2-cyclic components, and such that $f=u v$. For then, by interdigitating $m$-blocks of the 2-cyclic components of $u$, we obtain a permutation $a$ such that $C_{Z}(a)=C_{Z}(a ; 2 m)$, and such that $a^{m}=u$. And similarly we obtain $b$ such that $C_{Z}(b)=C_{Z}(b ; 2 n)$, and such that $b^{n}=v$. Hence $f=u v=a^{m} b^{n}=a^{M} b^{N} a^{m} b^{n}$, since $a^{M}=b^{N}=$ the identity permutation of $Z$. Therefore $x^{M} y^{N} x^{m} y^{n}$ is universal for $S_{Z}$, and hence is ISymuniversal.

Let $c_{k}$ denote the $k$-cycle $(01 \cdots k-1)$ in $Z$. We recall that $c_{k}(0 k-2)(1 k-3)$ $\cdots(z k-2-z)=(0 k-1)(1 k-2) \cdots(z k-1-z)$ where $z$ is the largest integer $i \leqslant(k-2) / 2$. Therefore, when $h$ is defined by $C_{Z}(h)=\{(k+2 i k+2 i+1)$ : $i \in \omega\}$, we have that

$$
\begin{aligned}
c_{k}= & c_{k} h^{2}=(0 k-1)(1 k-2) \cdots(z k-1-z) \\
& \cdot(z k-2-z)(z-1 k-1-z) \cdots(1 k-3)(0 k-2) h^{2} \\
= & H_{1} H_{2},
\end{aligned}
$$

where $H_{1}$ is the involution $(0 k-1)(1 k-2) \cdots(z k-1-z) h$ of $Z$, and where $H_{2}$ is the involution $(z k-2-z)(z-1 k-1-z) \cdots(1 k-3)(0 k-2) h$ of $Z$. We remark also that $s=H_{3} H_{4}$, where $H_{3}$ is the involution $(01)(-12) \cdots(-t t+1) \cdots$ of $Z$, and where $H_{4}$ is the involution $(-11)(-22) \cdots(-t t) \cdots$ of $Z$. It easily follows that, when $\{i: f(i)=i\}$ is infinite, then there exist involutions $u$ and $v$ of the desired sort. Thus we may suppose now that the set $\{i: f(i)=i\}$ is not infinite. There are two cases.

Case. $C_{Z}(f ; \omega) \neq \varnothing$. As a paradigm example take $\{(01),(234),(5678)\}$ to be the set of all finite cyclic components of $f$. Choose $g \in C_{Z}(f ; \omega)$. Then $g=H_{5} H_{6}$ for some involutions $H_{5}$ and $H_{6}$ of $Z$, each of which has infinitely many 2-cyclic components, and such that $Z £ H_{5} \cup Z £ H_{6} \subseteq Z £ g$. Observe also that

$$
\begin{aligned}
(01)(234)(5678) & =(01)(234)(5678)(23)^{2}(57)^{2} \\
& =(01)(234)(23)(5678)(57)(23)(57) \\
& =(01)(24)(58)(67) \cdot(23)(57),
\end{aligned}
$$


and hence that

$$
(01)(234)(5678) g=(01)(24)(58)(67) H_{5} \cdot(23)(57) H_{6} \text {. }
$$

The elements in $C_{Z}(f ; \omega) \backslash\{g\}$ pose no difficulty. It is now plain in the case that $C_{Z}(f ; \omega) \neq \varnothing$ that the desired involutions $u$ and $v$ exist.

Case. $C_{Z}(f ; \omega)=\varnothing$. Then $f$ has infinitely many cyclic components of finite length. Define $H_{7}$ by $C_{Z}\left(H_{7}\right)=C_{Z}(f ; 2)$, and define $H_{8}$ by

$$
C_{Z}\left(H_{8}\right)=\bigcup\left\{C_{Z}(f ; r): 2<r \in \omega\right\} .
$$

Then $f=H_{7} H_{8}=H_{8} H_{7}$. There exists an involution $H_{9}$ of $Z$ such that $H_{8} H_{9}$ also is an involution, and such that $Z £ H_{9} \subseteq Z £ H_{8}$. Clearly the sets $\left\{H_{7}, H_{8} H_{9}\right\}$ and $\left\{\mathrm{H}_{7}, \mathrm{H}_{9}\right\}$ are pdsp.

Subcase. $C_{Z}\left(H_{7}\right)$ is not infinite. Then both $C_{Z}\left(H_{8} H_{9}\right)$ and $C_{Z}\left(H_{9}\right)$ are infinite. Let $u=H_{7} H_{8} H_{9}$, and let $v=H_{9}$.

Subcase. $C_{Z}\left(H_{7}\right)$ is infinite. Then there exists a bijection $K: \omega \rightarrow C_{Z}\left(H_{7}\right)$ defined by $K: i \mapsto K_{i}$. Now define $H_{10}$ by $C_{Z}\left(H_{10}\right)=\left\{K_{2 i}: i \in \omega\right\}$, and define $H_{11}$ by $C_{Z}\left(H_{11}\right)=\left\{K_{2 i+1}: i \in \omega\right\}$. Let $u=H_{8} H_{9} H_{10}$, and let $v=H_{9} H_{11}$.

It is easy to see in both of these subcases that $u$ and $v$ are involutions of $Z$, each of which has infinitely many 2 -cyclic components, and such that $f=u v$.

We call $W$ a semigroup word iff all of the exponents of letters appearing in $W$ are positive. Of course every semigroup word is cyclically reduced.

By [8, Theorem 3.1], if $W$ is both semigroup and primitive, then there exists a semigroup word $V$ which is group-equivalent to $W$, and which is of the form $V=A B A$ only for $A$ the empty word and for $B=V$; such a word $V$ is called unbordered by $\mathrm{J}$. R. Isbell, who proves in [11] that if $X$ is infinite, and if $g \in{ }^{X} X$, then the equation $g=V$ is solvable in ${ }^{X} X$, where ${ }^{X} X$ denotes the semigroup of all transformations $h: X \rightarrow X$. Of course the symmetric group $S_{X}$ is a subgroup of the monoid ${ }^{X} X$. Thus we have

Proposition 19. If $W$ is both primitive and semigroup, then $W$ is group-equivalent to a word $V$ such that for every $f \in S_{Z}$ the equation $f=Y$ is solvable in ${ }^{z} Z$.

His proof of the cited Isbell theorem definitely does not produce a solution in $S_{Z}$ for the equation $f=V$ of Proposition 19. Nevertheless, Proposition 19 seems suggestive in the light of Proposition 3.

[6, Theorem 3] states that if $W$ is universal for $S_{X}$ for some infinite set $X$, then $W$ is universal for $S_{Y}$ for every uncountable set $Y$.

According to [6], the following two questions are open: If $W$ is universal for $S_{R}$, then is $W$ ISym-universal, where $R$ denotes the set of all real numbers? If $W$ is universal for every finite $S_{k}$, then is $W$ ISym-universal?

An affirmative answer to our titular question implies an affirmative answer to both of the questions cited in the preceding paragraph.

ACKNOWLEDGEMENTS. We are indebted to Klaus Leeb, Jan Mycielski and Milton Borba for their advice. 


\section{REFERENCES}

1. Jurema Arante, Sobre a ISym-universalidade de palavras primitivas, Dissertação de Mestrado, Universidade Federal de Santa Catarina, Florianópolis, Brasil, 1981.

2. E. A. Bertram, Permutations as products of conjugate infinite cycles, Pacific J. Math. 39 (1971), 275-284.

3.

, Even permutations as a product of two conjugate cycles, J. Combinatorial Theory Ser. A 12 (1972), 368-389.

4. M. P. Borba, D. M. Silberger and M. L. Valente, Representing the infinite cycle (to appear).

5. M. Droste and R. Göbel, On a theorem of Baer, Schreier, and Ulam for permutations, J. Algebra 58 (1979), 282-290.

6. A. Ehrenfeucht, S. Fajtlowicz, J. Malitz and J. Mycielski, Some problems on the universality of words in groups, Algebra Universalis 11 (1980), 261-263.

7. A. Ehrenfeucht and D. M. Silberger, Universal terms of the form $B^{n} A^{m}$, Algebra Universalis 10 (1980), 96-116.

8. Periodicity and unbordered segments of words, Discrete Math. 26 (1979), 101-109.

9. A. B. Gray, Infinite symmetric groups and monomial groups, Doctoral Dissertation, New Mexico State University, Las Cruces, New Mexico, 1960.

10. D. H. Husemoller, Ramified coverings of Riemann surfaces, Duke Math. J. 29 (1962), 167-174.

11. J. R. Isbell, On the problem of universal terms, Bull. Acad. Polon. des Sciences 14 (1966), 593-595.

12. R. J. Lyndon, Equations in groups, Bol. Soc. Brasil Mat. 11 (1980), 79-102.

13. Jan Mycielski, Can one solve equations in groups?, Amer. Math. Monthly 84 (1977), 723-726.

14. , Equations unsolvable in $G L_{2}(C)$ and related problems, Amer. Math. Monthly 85 (1978), 263-265.

15. O. Ore, Some remarks on commutators, Proc. Amer. Math. Soc. 2 (1951), 307-314.

16. M. L. Valente, Sobre a universalidade de palavras para grupos simétricos, Dissertação de Mestrado, Universidade Federal de Santa Catarina, Florianópolis, Brasil, 1979.

Departamento de Matemática, Universidade Federal de Santa Catarina, 88000-FlorianoPOLIS-SC-BRASIL

Current address: Department of Mathematics, Saint Martin’s College, Lacey, Washington 98503 